\title{
Impact of the formaldehyde concentration in the air on the sink effect of a coating material
}

\author{
Anne-Lise Tiffonnet ${ }^{1, *}$, Céline Tourreilles ${ }^{2}$, and Thierry Duforestel $^{2}$ \\ ${ }^{1}$ LUSAC, Université de Caen Normandie, Saint-Lô, France \\ ${ }^{2}$ EDF Département EnerBAT, EDF Lab Les Renardières, France
}

\begin{abstract}
This study aims to characterize, from a numerical modelling, the sorption behaviour of a material (a plasticised flooring material) when it is exposed to a pollutant commonly encountered in indoor environments (formaldehyde). It deals with the influence of the pollutant concentration in the room air on the sink effect of the material. The numerical simulations are based on a macroscopic modelling using experimental test results obtained elsewhere. The consequences on the room inertia are also discussed, and analogies between mass transfer and heat transfer are highlighted.
\end{abstract}

\section{Introduction}

In the fields of energy efficiency and indoor air quality of buildings, the characterisation of the thermal and solutal behaviour of the used construction and coating materials is essential. The prediction of the temperatures of the air and the walls of a room allows to evaluate the thermal comfort. At the same time, the prediction of the pollutants concentrations allows to appreciate the risks for the occupants' health.

When dealing with pollutant transfers between air phase and walls, many studies represent these transfers like global processes and walls are often considered as reversible sinks. Two coefficients called "adsorption rate constant" and "desorption rate constant" are used to describe respectively the pollutant transfer from de room air to the wall and from the wall to the room air.

This study deals with the influence of the concentration level of an indoor air pollutant (formaldehyde) on the sorption capacity of a material used as typical flooring material (plasticised flooring material).

\section{Description of the macroscopic model}

The most widely used model for predicting either the VOC emission from materials or the adsorption and desorption cycles of airborne contaminants is the onesink model initially suggested by Tichenor and coworkers [1]. It considers that at any time, the mass flux on the material surface is the difference between the adsorption and the desorption rates of the material. According to Langmuir's theory, the former is assumed to be proportional to the contaminant concentration in the bulk air of the room, while the latter is assumed to depend upon the amount of contaminant adsorbed on the material surface.
Therefore, the governing equations for the indoor concentration $C\left(\mathrm{~kg} \cdot \mathrm{m}^{-3}\right)$ and the surface mass $M\left(\mathrm{~kg} \cdot \mathrm{m}^{-2}\right)$ of a compound, respectively, are (fig. 1):

$$
\begin{aligned}
& V \frac{d C}{d t}=Q\left(C_{e x t}-C\right)-S k_{a} C+S k_{d} M \\
& \frac{d M}{d t}=k_{a} C-k_{d} M
\end{aligned}
$$

where $C_{\text {ext }}\left(\mathrm{kg} \cdot \mathrm{m}^{-3}\right)$ is the pollutant concentration in the inlet air, $V\left(\mathrm{~m}^{3}\right)$ the room volume, $Q\left(\mathrm{~m}^{3} \cdot \mathrm{s}^{-1}\right)$ the air exchange flow through the room, $k_{a}\left(\mathrm{~m} . \mathrm{s}^{-1}\right)$ the adsorption rate coefficient, $k_{d}\left(\mathrm{~s}^{-1}\right)$ the desorption rate constant, and $S\left(\mathrm{~m}^{2}\right)$ the material surface area exposed to the bulk air. It means that increased surface areas due to surface roughness and internal pores of the material, as well as the effects of bulk mass transport and diffusive transport processes, are implicitly lumped into the adsorption and desorption rate coefficients.

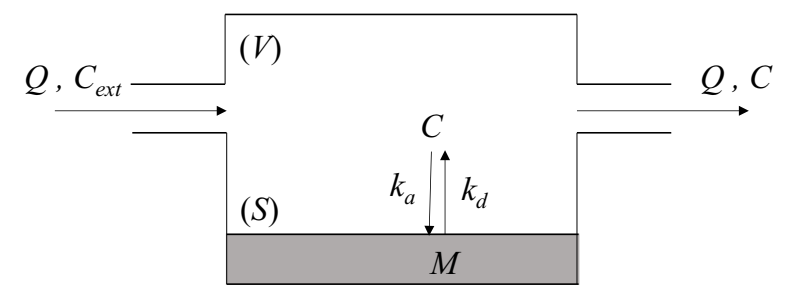

Fig. 1. Schematic representation of pollutant transfers.

\section{Sorption capacity of a material}

\subsection{Experimental characterisation}

In order to characterize the sorption capacity of the studied material, tests in experimental chamber were carried out.

* Corresponding author: anne-lise.tiffonnet@unicaen.fr 
The experimental device is based on an existing equipment previously used to characterize sorption phenomena by static experiments [2]. This equipment was then adapted to characterize the same phenomena by dynamic experiments. This experimental device is described in details elsewhere [3].

Once the material is placed in the chamber and the climate parameters (temperature and humidity) are stabilised, a known amount of pollutant is injected in the supply air of the chamber. The polluted air then flows continuously during the adsorption test period. Then, the comparison between the pollutant concentration in the supply air of the chamber and the exhaust one leads to determine the pollutant mass which is adsorbed by the material.

Once the steady state is reached (i.e. when we cannot observe any variation of the concentration in the air of the chamber), the pollutant injection is stopped. The supply air of the chamber is now a clean air. This is the desorption phase. Comparing the profiles of the obtained concentrations with and without material allows to determine the pollutant mass which is desorbed by the material.

Experiments were carried out with a plasticised flooring material exposed to formaldehyde under dry air (measured relative humidity is $8 \%$ ) at a constant temperature equals to $19^{\circ} \mathrm{C}$. The pollutant injection was imposed during 3 hours (adsorption phase). Measurements were realised continuously during 6 hours (adsorption + desorption periods) with sampling points every 3 minutes. The loading factor of the material (i.e. the ratio between the material area exposed to the room air and the chamber volume) was $0.628 \mathrm{~m}^{-1}$. Figure 2 shows the profile of the resulting concentration in the chamber air. For this test, the injection corresponded to an equilibrium concentration of $174 \mu \mathrm{g} \cdot \mathrm{m}^{-3}$. Without material, concentration would be comparable to the injection scheme one.

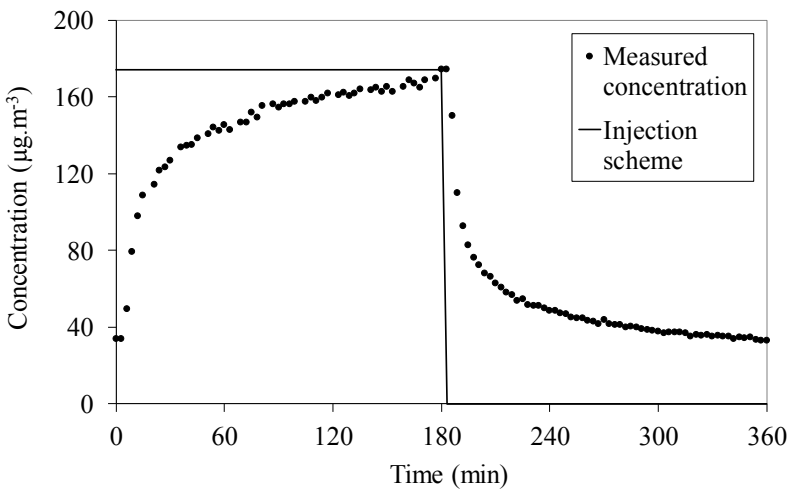

Fig. 2. Injection scheme and resulting concentration profile in the chamber air.

\subsection{Identification of the model coefficients}

This part presents the method used for the $k_{a}$ and $k_{d}$ coefficients identification. The principle is explained from the results of the experiment described above.

The insertion of equation (2) in equation (1) leads to, with $C_{\text {ext }}=C_{\infty}$ : $\frac{d C}{d t}=\frac{Q}{V} C_{\infty}-\frac{Q}{V} C-\frac{S}{V} \frac{d M}{d t}$

The gaseous phase concentration variation is calculated by using the following finite differences scheme:

$$
\frac{d C}{d t}=\frac{C^{t+\Delta t}-C^{t}}{\Delta t}
$$

Where $C^{t}$ is the concentration at the time $t, C^{t+\Delta t}$ is the concentration at the next time and $\Delta t$ is the time lapse.

The mass variation within the material is, from equation (3):

$$
\frac{d M}{d t}=-\frac{V}{S}\left(\frac{d C}{d t}+\frac{Q}{V}\left(C-C_{\infty}\right)\right)
$$

Here it is important to note that the concentration $C_{\infty}$ is the steady state concentration (i.e. the concentration which is reached at the end of the adsorption phase) in these equations. The pollutant mass inside the material is calculated any time by the equation (6) with, as the initial condition, a null initial mass in the sink (material): $M_{0}=0$.

$$
M^{t+\Delta t}=\left(\frac{d M}{d t}\right) \Delta t+M^{t}
$$

where $M^{t}$ is the mass in the sink at time $t$, and $M^{t+\Delta t}$ is the mass at the next stage.

Equation (2) can be rewritten as such:

$$
\left(\frac{d M}{d t}\right) / M=k_{a} C / M-k_{d}
$$

It is then possible to graphically determine the $k_{a}$ and $k_{d}$ coefficients from the draw of the following function:

$$
\left(\frac{d M}{d t}\right) / M=f(C / M)
$$

The coefficients of the straight line in the transient state give directly the $k_{a}$ and $k_{d}$ coefficients, as shown in figure 3. Thus we obtained for the adsorption phase: $k_{a}=$ $8.6510^{-2} \mathrm{~m}^{-1}{ }^{-1}$ et $k_{d}=9.0010^{-4} \mathrm{~s}^{-1}$. These values allow to compute the concentration and to compare it to the measured one (fig. 4).

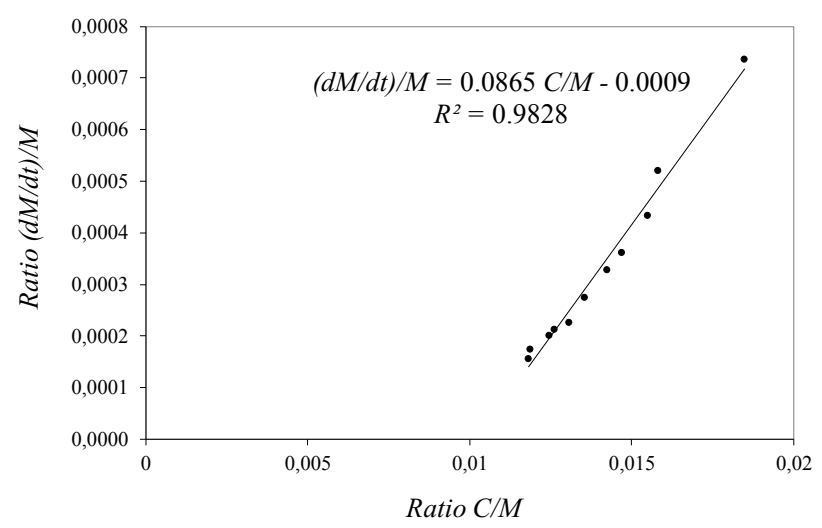

Fig. 3. Identification of $k_{a}$ and $k_{d}$ coefficients. 


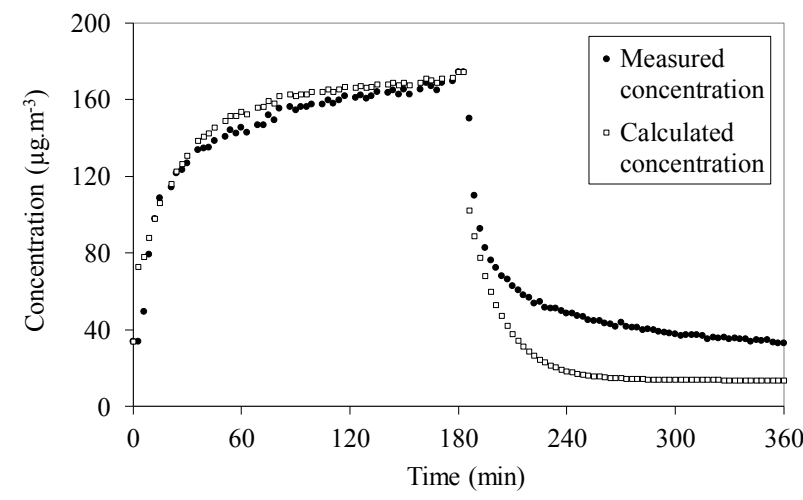

Fig. 4. Profiles of the measured and calculated chamber air concentrations.

The $k_{a}$ and $k_{d}$ values relevance is checked recovering the following relation in steady state:

$$
k_{a} C_{\infty}=k_{d} M_{\infty} \quad \text { or } \quad \frac{k_{a}}{k_{d}}=\frac{M_{\infty}}{C_{\infty}}
$$

It is checked here because the same value is found for the two ratios $(96,1 \mathrm{~m})$.

Theoretically, the $" k_{a}-k_{d}$ » model being based on total reversibility of the phenomena, the coefficients must be the same for the two phases (adsorption and desorption). The drawn in fig. 4 leads to the same conclusions as Singer and co-workers [4]: there is a larger difference between the measured and calculated concentration values for the desorption period than the adsorption period, coefficients being determined from the adsorption period.

To characterize the desorption period with other values of $k_{a}$ and $k_{d}$ coefficients, these values must check the equality (9). The values are obtained by iterative calculations. For the studied case, the coefficients are: $k_{a}$ $=4.8810^{-3} \mathrm{~m} \cdot \mathrm{s}^{-1}$ and $k_{d}=3.3010^{-4} \mathrm{~s}^{-1}$. The new drawn of the evolution of the computed concentration is given figure 5. Once again, a difference between measured values and computed values is observed for desorption period, but it is lower than previously. These observations match those by Singer and co-workers [4].

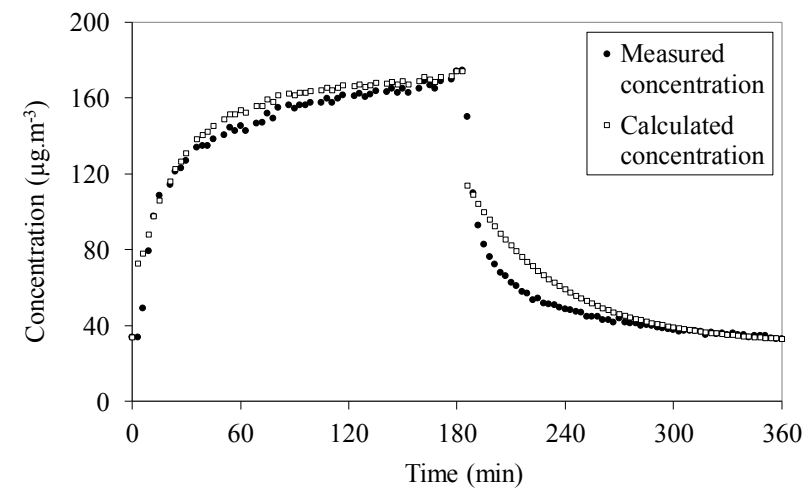

Fig. 5. Profiles of the measured and calculated chamber air concentrations with two different $« k_{a}-k_{d} »$ values.
Other tests were carried out in the experimental chamber with the same environmental conditions (same temperature, same relative humidity) and the same material loading factor but for two other equilibrium concentration levels $\left(71\right.$ and $\left.118 \mu \mathrm{g} \cdot \mathrm{m}^{-3}\right)$. The durations of the injection and desorption periods were also the same as previously ( 3 hours for each one). The exploitation of these tests, as previously explained, leads to obtain other $k_{a}$ and $k_{d}$ values.

Table 1 gives all the obtained values, which match data from literature $[1,4,5,6,7,8]$.

Table 1. Values of the model coefficients.

\begin{tabular}{|c|c|c|c|c|}
\cline { 2 - 5 } \multicolumn{1}{c|}{} & \multicolumn{2}{c|}{ Adsorption } & \multicolumn{2}{c|}{ Desorption } \\
\hline $\begin{array}{c}C \\
\left(\mu \mathrm{g} . \mathrm{m}^{-3}\right)\end{array}$ & $\begin{array}{c}k_{a} \\
\left(\mathrm{~m}^{-1} \mathrm{~s}^{-1}\right)\end{array}$ & $\begin{array}{c}k_{d} \\
\left(\mathrm{~s}^{-1}\right)\end{array}$ & $\begin{array}{c}k_{a} \\
\left(\mathrm{~m}_{\mathrm{s}}{ }^{-1}\right)\end{array}$ & $\begin{array}{c}k_{d} \\
\left(\mathrm{~s}^{-1}\right)\end{array}$ \\
\hline 71 & $6.8410^{-2}$ & $1.7010^{-3}$ & $1.2210^{-2}$ & $3.3010^{-4}$ \\
\hline 118 & $9.5810^{-2}$ & $1.3010^{-3}$ & $1.6910^{-2}$ & $5.3010^{-4}$ \\
\hline 174 & $8.6510^{-2}$ & $0.9010^{-3}$ & $4.8810^{-3}$ & $3.3010^{-4}$ \\
\hline
\end{tabular}

No conclusion can be drawn from these results. To compare them, it will be necessary and even required to neglect: 1) the fact that initial concentrations are not null in adsorption phase, and 2) the fact that the steady-state concentrations are not null in desorption phase. Thus, a dimensionless concentration is defined and used thereafter.

\section{Results exploitation}

\subsection{Reduced concentration}

Like the reduced temperature occurs in heat transfer, the reduced concentration is defined by the following expression:

$$
C^{*}=\frac{C(t)-C_{\infty}}{C_{0}-C_{\infty}}
$$

where $C_{0}$ is the initial concentration and $C_{\infty}$ is the steady state concentration (equilibrium concentration in the room air).

The value of this dimensionless concentration ranges from 0 to 1 . The closer it is to 1 , the closer the concentration is to the initial concentration. It means a total adsorption of the injected pollutant. Or rather, the closer the reduced concentration is to 0 , the closer the concentration is to the injected one. In this case, it means that the adsorption is very low.

Figure 6 shows the reduced concentration evolutions for the three studied concentration levels (formaldehyde injection concentrations: 174,118 , et $71 \mu \mathrm{g} . \mathrm{m}^{-3}$ ) during the adsorption phase. The fact of being able to distinguish the three curves indicates a dependence on the pollutant concentration of the sorption capacity of the material. One can see that the adsorption capacity seems to be growing up along with the concentration. 


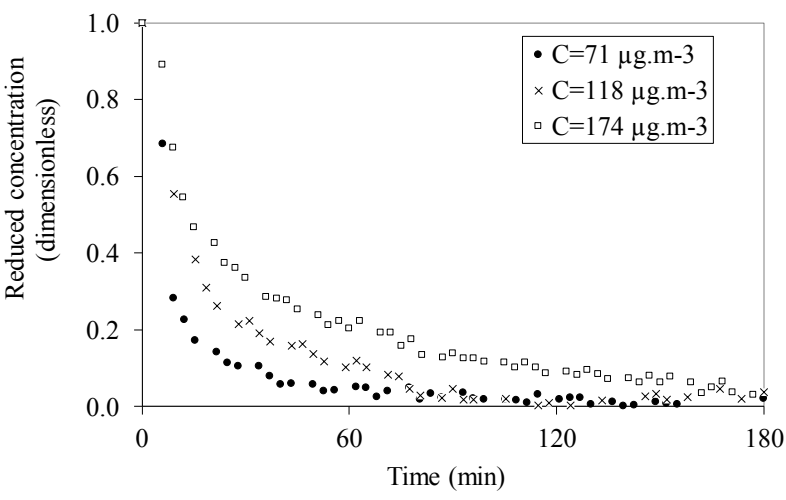

Fig. 6. Profile of the reduced concentration during the adsorption period, for the three studied concentration levels.

Equation (1) can be rewritten as:

$$
\frac{d C}{d t}+\left(\frac{Q}{V}+k_{a} \frac{S}{V}\right) C=\frac{Q}{V} C_{\infty}+k_{d} \frac{S}{V} M
$$

The solution to this equation without second term takes the following form:

$$
C=A \exp \left(-\left(\frac{Q}{V}+k_{a} \frac{S}{V}\right) t\right)
$$

A specific solution, corresponding to the steady state, is, with $d C / d t=0$ :

$$
C=\frac{\frac{Q}{V} C_{\infty}+k_{d} \frac{S}{V} M_{\infty}}{\left(\frac{Q}{V}+k_{a} \frac{S}{V}\right)}=C_{\infty}
$$

Thus, the solution to equation (11) is the sum of the two previous results:

$$
C=A \exp \left(-\left(\frac{Q}{V}+k_{a} \frac{S}{V}\right) t\right)+C_{\infty}
$$

where $A$ is determined from the initial condition of the adsorption phase: $A=C_{0}-C_{\infty}$.

Consequently, the reduced concentration profile can be expressed as:

$$
C^{*}=\exp \left(-\left(\frac{Q}{V}+k_{a} \frac{S}{V}\right) t\right)
$$

or:

$$
C^{*}=\exp \left(-\frac{t}{\tau}\right)
$$

where $\tau$ is the time constant (s).

Here, the analogy with heat transfer is highlighted. In the case of thermally thin media within which temperature is considered as uniform, the evolution of the reduced temperature is given exactly by the same expression.

When dealing with the desorption period, equation (1) can be rewritten as follows (with $\mathrm{C}_{e x t}=0$ ):

$$
\frac{d C}{d t}+\left(\frac{Q}{V}+k_{a} \frac{S}{V}\right) C=k_{d} \frac{S}{V} M
$$

As previously, the form of the solution of the equation without second term is given by expression (12). When the steady state is reached, the room air concentration would be equal to the background level (or initial concentration of the adsorption period). At the beginning of the desorption period the concentration is the concentration reached at the end of the adsorption period. The integration constant is obtained from this initial condition: $A=C_{\infty}-C_{0}$.

Thus, the reduced concentration profile takes the form of the following expression:

$$
C^{*}=1-\exp \left(-\left(\frac{Q}{V}+k_{a} \frac{S}{V}\right) t\right)
$$

\subsection{Time constant and inertia}

The time constant of the phenomenon, $\tau$, is such as:

$$
\frac{1}{\tau}=\frac{Q}{V}+k_{a} \frac{S}{V} \quad \text { or } \quad \tau=\frac{V}{Q+k_{a} S}
$$

It is possible to evaluate this constant by drawing the function $\operatorname{Ln}\left(C^{*}\right)=f(t)$.

The time constant values fo the three studied concentration levels and obtained from the adsorption period are indicated in table 2. Time constant increases as concentration increases. For a given loading factor, the higher the concentration is, the longer the time to reach equilibrium is.

The time constant is characteristic of inertia. The higher the time constant is, the larger inertia is. A material is considered as thermally inert when the energy it can store during a time lapse is significant. Otherwise it is qualified as reactive.

In the same way, a material would be considered as a pollutant buffer if the mass it could adsorb is significant. The profile of the mass in the sink for the three studied concentration levels is presented in figure 7. The resulting adsorbed masses during the adsorption periods were computed from the time integration of the solution of equation (2).

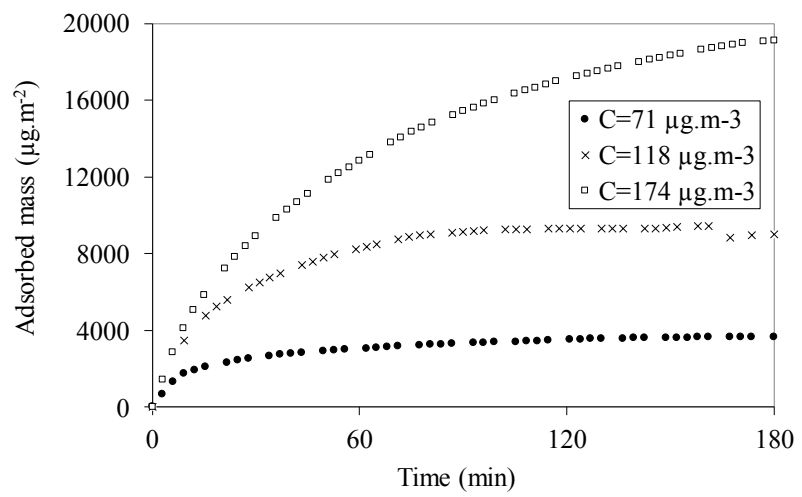

Fig. 7. Evolution of the adsorbed mass on the sink during the adsorption period, for the three concentration levels. 
Equation (2) can be rewritten:

$$
\frac{d M}{d t}+k_{d} M=k_{a} C
$$

The form of the solution of the same equation without second term is:

$$
M=B \exp \left(-k_{d} t\right)
$$

and a specific solution corresponding to the equilibrium is: $M=M_{\infty}$.

Elsewhere, the constant is determined from the initial condition of the adsorption period: $B=-M_{\infty}$. Thus:

$$
M=M_{\infty}\left(1-\exp \left(-k_{d} t\right)\right)
$$

The time integration of this last equation leads to the total mass $(\mathrm{m})$ of pollutant adsorbed during the time of the adsorption period. The values for the three studied levels of pollutant concentration are also given in table 2 . These results confirm the previous conclusions.

Table 2. Time constant and adsorbed mass.

\begin{tabular}{|c|c|c|}
\hline$C\left(\mu \mathrm{g} \cdot \mathrm{m}^{-3}\right)$ & $\tau(\mathrm{min})$ & $m(\mu \mathrm{g})$ \\
\hline 71 & 12.05 & 78.5 \\
\hline 118 & 18.87 & 234.3 \\
\hline 174 & 22.22 & 434.3 \\
\hline
\end{tabular}

\section{Conclusion}

Apart from the notion of reduced concentration analogous to the reduced temperature one - there are many analogies between heat and mass transfers [9]. Notions like time constant and inertia are analogous: the more a material occurs as a wall component of the room the more it gives inertia to this room. The larger the material surface is exposed to the room air, the more important the sink effect due to the material is: the room air concentration is smaller during the adorption period and the pollutant reemission is slower during the desorption period.

Here we studied a synthetic non-porous material, and we used a $" k_{a}-k_{d}$ » model which is based on the hypothesis that concentrations on the material surface and room air are in equilibrium. Transfers inside the materials were neglected. This modelling is analogous to the heat transfer one for thin material - which does not display any temperature gradient (temperature is uniform) - exposed to an external temperature variation.

This simplification leads to some discrepancies between the measured data (results from experimental tests) and the computed one (results from the model). The $k_{a}$ and $k_{d}$ coefficients would depend on the material and the air flow on the surface of this material.
It also depends on the pollutant transfers (mainly diffusion [10]) which take place within the material after the adsorption or before the desorption. Thus, the knowledge of the solubility constant for the studied pollutant/material couple and the diffusion coefficient of the pollutant within the material would be useful in order to evaluate the lack of precision of the simplified model which has been used here.

We wish to thank the laboratory LaSIE of the University of La Rochelle for the use and the exploitation of the experimental results.

\section{References}

1. B. Tichenor, Z. Guo, J. Dunn, L. Sparks, M. Mason. The Interaction of Vapor Phase Organic Compounds with Indoor Sinks. Indoor Air, 1, pp. 23-35, (1991)

2. A.L. Tiffonnet. Contribution à l'analyse de la Qualité de l'Air Intérieur : Influence des transports de Composés Organiques Volatils (COV) entre les parois et l'ambiance. $\mathrm{PhD}$ thesis - Université de La Rochelle (2000)

3. C. Tourreilles. Qualification énergétique et sanitaire des systèmes d'épuration intégrés aux réseaux de ventilation. PhD thesis - Université de La Rochelle (2015)

4. B. Singer, A. Hodgson, K. Guevarra, E. Hawley, W. Nazaroff. Gas-phase organics in environmental tobacco smoke. Effects of smoking rate, ventilation, and furnishing level on emission factors. Environmental Science \& Technology, 36, pp. 846-853, (2002)

5. Y. An, J. Zhang, C Shaw. Measurements of VOC adsorption / desorption characteristics of typical interior building materials. HVAC\&R Research : International Journal of Heating, Ventilating, Air-Conditioning and Refrigerating Research, 5, issue 4, pp. 297-316, (1999)

6. W. Bouhamra, A. Elkilani. Investigation and modeling of surface sorption / desorption behavior of volatile organic compounds for indoor air quality analysis. Environmental Technology, 20, issue 5, pp. 31-45, (1999)

7. R. Jorgensen, T. Dokka, O. Bjorseth. Introduction of a Sink-Diffusion Model to Describe the Interactions between Volatile Organic Compounds (VOCs) and Material Surfaces. Indoor Air; 10, pp. 27-38, (2000)

8. D. Won, R. Corsi, M. Rynes. Sorptive Interactions between VOCs and Indoor Materials. Indoor Air, 11, pp. 246-256, (2001)

9. A. Damian, A.L. Tiffonnet, P. Blondeau, F. Allard. Mise en évidence de l'effet des parois sur les concentrations intérieures en polluants - Analogie avec le transfert thermique, Actes du Forum des Associations du Génie Civil et Urbain, Lyon, pp. 125-132, (2000)

10. P. Blondeau, A.L. Tiffonnet; F. Allard, F. Haghighat. Physically-based modeling of the material and gaseous contaminant interactions in buildings: models, experimental data and future developments. Advances in Building Energy Research, 2, pp. 57-94, (2008) 